\title{
Giant Congenital Melanocytic Nevus Accompanied by an Intracranial Arachnoid Cyst
}

\author{
Sara Dusel ${ }^{1}$, Nina Trenkler ${ }^{1}$, Christine Fink ${ }^{1}$, Ferdinand Toberer ${ }^{1}$, Sven Krengel ${ }^{2}$, \\ Alexander Enk, ${ }^{1}$ Holger A. Haenssle ${ }^{1}$
}

\begin{abstract}
1 Department of Dermatology, Venerology, and Allergology; University Medical Center, Ruprecht-Karls University, Heidelberg, Germany 2 Dermatologische Gemeinschaftspraxis, Lubeck, Germany
\end{abstract}

Key words: giant congenital melanocytic nevus, arachnoid cyst, neurocutaneous melanocytosis, melanoma risk

Citation: Dusel S, Trenkler N, Fink C, Toberer F, Krengel S, Enk A, Haenssle HA. Giant congenital melanocytic nevus accompanied by an intracranial arachnoid cyst. Dermatol Pract Concept. 2019;9(3):225-227. DOI: https://doi.org/10.5826/dpc.0903a14

Accepted: February 8, 2019; Published: July 31, 2019

Copyright: $\odot 2019$ Dusel et al. This is an open-access article distributed under the terms of the Creative Commons Attribution License, which permits unrestricted use, distribution, and reproduction in any medium, provided the original author and source are credited.

Funding: None.

Competing interests: The authors have no conflicts of interest to disclose.

Authorship: All authors have contributed significantly to this publication.

Corresponding author: Holger A. Haenssle, MD, Department of Dermatology, Venerology and Allergology, University Medical Center, Ruprecht-Karls-University, Im Neuenheimer Feld 440, 69120 Heidelberg, Germany. Email: holger.haenssle@med.uni-heidelberg.de

\section{Introduction}

Owing to a somatic NRAS mutation at the stage of neural crest formation, patients with congenital melanocytic nevi (CMN) may have coexisting central nervous system (CNS) abnormalities. This risk of CNS abnormalities is higher in CMN with multiple satellite nevi [1]. Frequent CNS abnormalities include melanin deposits, melanocytic proliferations (neurocutaneous melanocytosis [NCM]), Dandy-Walker malformation, and spinal or cranial arachnoid cysts. Moreover, $\mathrm{CMN}$ with a diameter $>40 \mathrm{~cm}$ projected adult size are classified as giant congenital melanocytic nevi (GCMN) and carry an increased melanoma lifetime risk.

\section{Case Presentation}

We report a 33-year-old male patient with a GCMN covering his lower trunk and upper legs accompanied by large numbers of disseminated and partially hypertrichotic "satellite" nevi (Figure 1, A-D). There was a history of seizures at the age of 3 years, but no medical reports were available. The patient's current neurological status was unremarkable. Magnetic resonance imaging (MRI) scans of the brain and spinal cord revealed a large arachnoid cyst in the posterior cranial fossa (Figure 1, E-G). The cerebellum appeared compressed but no structural aberrations suggestive of a Dandy-Walker malformation were found (Figure 1, E-G). The brain parenchyma and leptomeninges showed no melanocytic proliferations.

We scheduled the patient for dermatological examinations every 6 months including sequential total body imaging, dermoscopy, and palpation of skin and lymph nodes. We recommended follow-up MRI scans of the brain and spine at 2-year intervals to monitor the arachnoid cyst and to detect any new CNS abnormalities. The patient was instructed to present immediately in case of any new neurological symptoms.

\section{Conclusions}

CNS involvement in CMN was termed neurocutaneous melanocytosis (NCM) and has long been assigned an unfavorable prognosis. Recent observations showed a dichotomy between "classic" NCM (showing widespread leptomeningeal involvement) and other CNS manifestations, including focal melanin deposits of the brain parenchyma and cysts [1]. Importantly, 

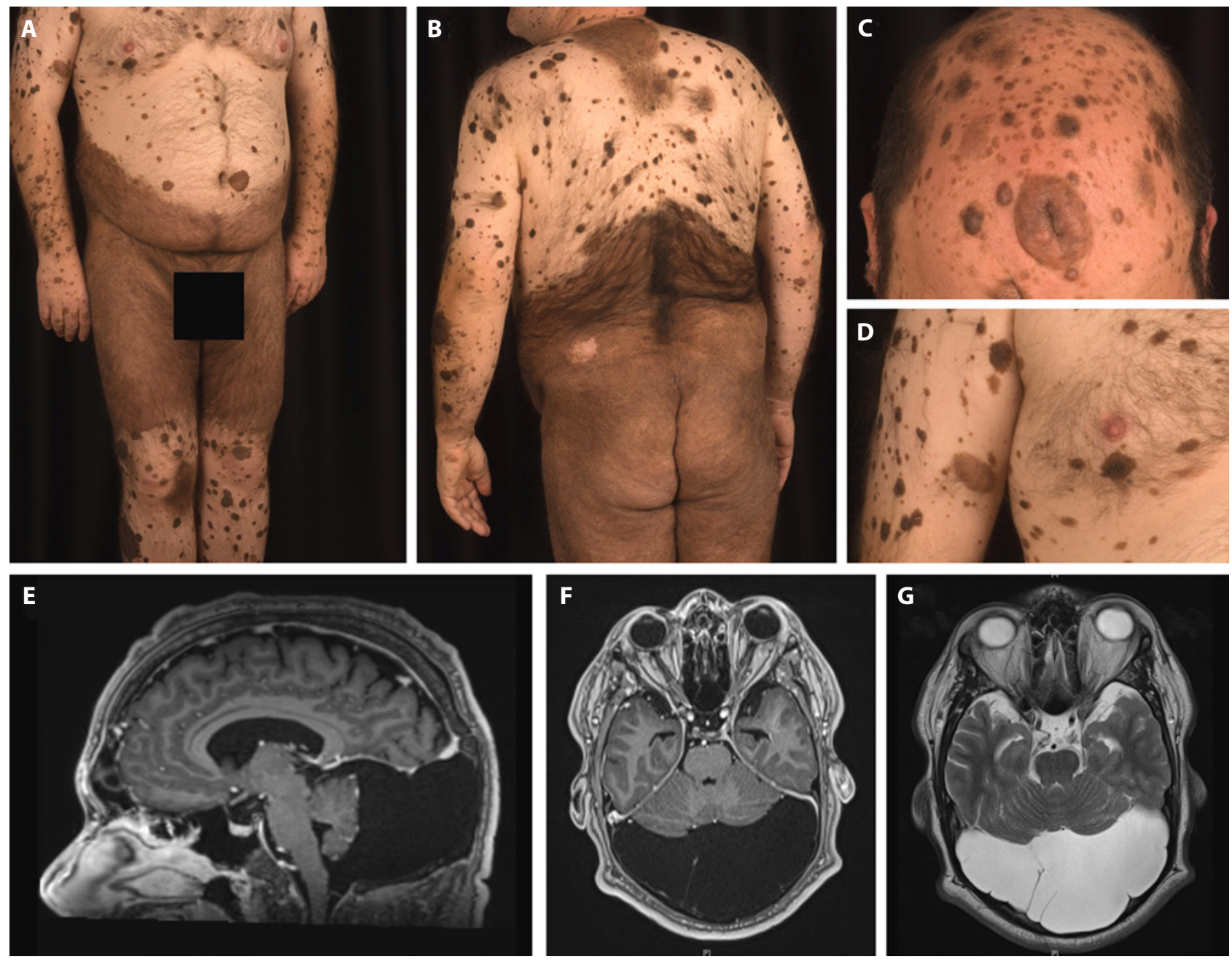

Figure 1. Clinical presentation and MRI scans of the brain. The lower trunk and both upper legs are covered by a GCMN (A). A second large melanocytic lesion is situated on the thoracic posterior midline (B). Many accompanying heterogeneous, partially hypertrichotic and nodular "satellite" nevi are spread over the whole body (C, D). Sagittal T1-weighted magnetization-prepared rapid gradient-echo (MP-RAGE) MRI of the brain revealed a large arachnoid cyst in the posterior cranial fossa (E). Axial T1-weighted MP-RAGE MRI scan of the brain (F) and axial T2-weighted turbo spin-echo (TSE) MRI of the brain (G) showed compression of the cerebellum but no structural aberrations. [Copyright: C2019 Dusel et al.]

in the case of "classic" NCM the risk of severe complications is considerably higher. Arachnoid cysts are frequent incidental findings in CNS imaging and for the most part do not require any medical intervention. It has been hypothesized that leptomeningeal melanosis may interfere with the formation of cere- brospinal fluid resorption pathways, resulting in the formation of posterior fossa or spinal arachnoid cysts. Table 1 lists previous publications reporting the rare association of GCMN with or without NCM and cerebral or spinal arachnoid cysts. However, in our patient MRI scans revealed no melanocytic

\section{Table 1}

\begin{tabular}{|l|l|}
\hline Author, Year [Reference] & \multicolumn{1}{c|}{ Observation } \\
\hline Frieden et al, 1994 [2] & $\begin{array}{l}\text { Case series of 20 neurologically asymptomatic patients with GCMN; brain abnormalities in } \\
\text { 9 patients, 1-month-old girl without NCM but left middle cranial fossa arachnoid cyst }\end{array}$ \\
\hline Foster et al, 2001 [3] & $\begin{array}{l}\text { Case series of 46 neurologically asymptomatic patients with GCMN; brain abnormalities in } \\
\text { 14 patients, 1 patient without signs of NCM by MRI but middle cranial fossa arachnoid cyst }\end{array}$ \\
\hline Holmes et al, 2001 [4] & $\begin{array}{l}\text { 43-year-old woman with GCMN and increasing thoracic pain, MRI without signs of NCM } \\
\text { but spinal arachnoid cyst }\end{array}$ \\
\hline Acosta et al, 2005 [5] & 5-month-old girl, GCMN, NCM, symptomatic hydrocephalus, spinal arachnoid cyst \\
\hline Ramaswamy et al 2012 [6] & $\begin{array}{l}\text { Case series of 14 patients with GCMN and NCM; 3 patients had extensive dorsal spinal } \\
\text { arachnoid cysts }\end{array}$ \\
\hline
\end{tabular}


deposits of leptomeninges [2-6]. Further studies are needed to establish whether there is a true pathogenic association between GCMN and arachnoid cysts. In a large, prospective cohort published by Kinsler et al [7], the risk of melanoma (CNS or cutaneous) was $12 \%$ in the group of children with an abnormal baseline MRI, compared with $2 \%$ in those with an unremarkable MRI. When stratified according to the largest diameter of the cutaneous nevus, the melanoma risk was $8 \%$ for $\mathrm{CMN}>60 \mathrm{~cm}$ and $1 \%$ for $\mathrm{CMN}$ of all other sizes.

MRI screening is justified in infants with $\mathrm{CMN}>40 \mathrm{~cm}$ in diameter and/or $>20$ satellite nevi at birth. The clinical management of CMN patients with symptomatic CNS abnormalities includes neurosurgery, anticonvulsant therapy, and shunt placement in cases of increased intracranial pressure. In general, problematic melanocytic lesions in children include large congenital nevi and Spitz nevi that may be viewed as either potential melanoma precursors or melanoma simulators, respectively [8]. Recommendations for management of the CMN and satellite nevi itself should incorporate the risk of malignant transformation as well as psychological implications of any disfigurement. Because of the limited benefit of any prophylactic excisions for melanoma prevention, dermatological follow-up examinations alone are a valuable option in many cases.

\section{References}

1. Waelchli R, Aylett SE, Atherton DJ, Thompson DJ, Chong WK, Kinsler VA. Classification of neurological abnormalities in children with congenital melanocytic naevus syndrome identifies magnetic resonance imaging as the best predictor of clinical outcome. $\mathrm{Br} \mathrm{J}$ Dermatol. 2015;173(3):739-750.

2. Frieden IJ, Williams ML, Barkovich AJ. Giant congenital melanocytic nevi: brain magnetic resonance findings in neurologically asymptomatic children. J Am Acad Dermatol. 1994;31(3):423-429.

3. Foster RD, Williams ML, Barkovich AJ, Hoffman WY, Mathes SJ, Frieden IJ. Giant congenital melanocytic nevi: the significance of neurocutaneous melanosis in neurologically asymptomatic children. Plast Reconstr Surg. 2001;107(4):933-941.

4. Holmes G, Wines N, Ryman W. Giant congenital melanocytic naevus and symptomatic thoracic arachnoid cyst. Australas J Dermatol. 2001;42:124-128.

5. Acosta FL Jr, Binder DK, Barkovich AJ, Frieden IJ, Gupta N. Neurocutaneous melanosis presenting with hydrocephalus: case report and review of the literature. J Neurosurg. 2005;102(1 Suppl):96-100.

6. Ramaswamy V, Delaney H, Haque S, Marghoob A, Khakoo Y. Spectrum of central nervous system abnormalities in neurocutaneous melanocytosis. Dev Med Child Neurol. 2012;54(6):563-568.

7. Kinsler VA, O'Hare P, Bulstrode N, et al. Melanoma in congenital melanocytic naevi. Br J Dermatol. 2017;176(5):1131-1143.

8. Moscarella E, Piccolo V, Argenziano G, et al. Problematic lesions in children. Dermatol Clin. 2013;31(4):535-547. 